\title{
Caracterização dos cursos de harmonização orofacial ofertados em um município do estado do Paraná
}

\author{
Characterization of the orofacial harmonization courses offered in a city in the state of Paraná \\ Caracterización de los cursos de armonización orofacial ofrecidos en una ciudad del estado de
}

Paraná

Recebido: 13/12/2021 | Revisado: 22/12/2021 | Aceito: 28/12/2021 | Publicado: 29/12/2021

\author{
Gisselly Maria Campos da Silva \\ ORCID: https://orcid.org/0000-0003-0995-8461 \\ Universidade Estadual de Maringá, Brasil \\ E-mail: gisselly_campos@hotmail.com \\ Vanessa Cristina Veltrini \\ ORCID: https://orcid.org/0000-0003-1343-9269 \\ Universidade Estadual de Maringá, Brasil \\ E-mail: vanessaveltrini@gmail.com \\ Giselma Leite dos Santos \\ ORCID: https://orcid.org/0000-0002-6562-2703 \\ Universidade Estadual de Maringá, Brasil \\ E-mail: giselma.leite88@gmail.com \\ Isabela Regina Grilo Silva \\ ORCID: https://orcid.org/0000-0001-7330-0651 \\ Universidade Estadual de Maringá, Brasil \\ E-mail: isabela.rgrilo@gmail.com \\ Luiz Fernando Lolli \\ ORCID: https://orcid.org/0000-0001-7426-5763 \\ Universidade Estadual de Maringá, Brasil \\ E-mail: lflolli@uem.br \\ Mitsue Fujimaki \\ ORCID: https://orcid.org/0000-0002-7824-3868 \\ Universidade Estadual de Maringá, Brasil \\ E-mail: mfujimaki@uem.br
}

\begin{abstract}
Resumo
O objetivo do trabalho foi descrever as características dos cursos de harmonização orofacial ofertados a cirurgiõesdentistas em um município da região Noroeste do Paraná. Trata-se de um estudo observacional, descritivo e transversal em que os dados foram coletados a partir da busca de informações em sites de instituições de ensino, em redes sociais e na plataforma Google, com as seguintes palavras-chave: "curso de" + "harmonização" ou "harmonização facial" ou "harmonização orofacial" ou "toxina botulínica" ou "botox" ou "preenchimento" ou "ácido hialurônico", limitando para uma cidade. Vinte cursos foram encontrados. As finalidades terapêuticas e estéticas dos procedimentos constavam nos conteúdos programáticos de $85 \%$ dos cursos, enquanto em $15 \%$ havia apenas a menção aos benefícios estéticos. $75 \%$ dos cursos se denominavam imersão, 15\% eram especializações, e $10 \%$ eram aperfeiçoamentos. 85\% informaram carga-horária e, em 73,68\% destes, a duração era menor ou igual a cinco dias. $\mathrm{O}$ valor da hora/aula variou de $\mathrm{R} \$ 65,00$ a $\mathrm{R} \$ 1.000,00$. Conclui-se que os cursos de harmonização orofacial ofertados apresentam alto valor, comparados aos cursos de outras especialidades, e são, em sua maioria, de curta duração. Além disso, verificou-se a necessidade das entidades promotoras adequarem o tipo de curso (atualização, aperfeiçoamento, especialização, dentre outros) à respectiva carga horária mínima exigida.
\end{abstract}

Palavras-chave: Odontologia; Estética; Ensino.

\begin{abstract}
The objective of this work was to describe the characteristics of orofacial harmonization courses offered to dentists in a city in the Northwest region of Paraná. This is an observational, descriptive and cross-sectional study in which data were collected from information searches on websites of educational institutions, social networks and the Google platform, with the following keywords: "course" + " matching" or "facial matching" or "orofacial matching" or "botulinum toxin" or "botox" or "filling" or "hyaluronic acid", limiting the search to a city. This resulted in 20 courses, whose therapeutic and aesthetic purposes of the procedures were included in the syllabus of $85 \%$ of the courses, while in $15 \%$ there were only aesthetic objectives. $75 \%$ of the courses were called immersion, $15 \%$ were specializations, while $10 \%$ were improvements. $85 \%$ reported working hours and, in $73.68 \%$ of these, the duration
\end{abstract}


was less than or equal to five days. The amount of hours/class charged to students ranged from $\mathrm{R} \$ 65.00$ to $\mathrm{R} \$ 1,000.00$. It is concluded that the orofacial harmonization courses offered are of high value, compared to courses in other specialties, and are mostly of short duration. In addition, there was a need for the entities promoting the courses to be alerted to the adequacy of the name or type (updating, perfecting, specialization, among others) to the minimum workload required.

Keywords: Dentistry; Esthetics; Teaching.

\section{Resumen}

El objetivo de este estudio fue describir las características de los cursos de armonización orofacial ofrecidos a los cirujanos dentistas en una ciudad de la región noroeste de Paraná. Se trata de un estudio observacional, descriptivo y transversal en el que los datos se recogieron a partir de la búsqueda de información en páginas web de instituciones educativas, redes sociales y plataforma Google, con las siguientes palabras clave: "curso" + "armonización" o "armonización facial" o "armonización orofacial" o "toxina botulínica" o "botox" o "relleno" o "ácido hialurónico", limitándose a una ciudad. Se encontraron 20 cursos. Los fines terapéuticos y estéticos de los procedimientos estaban incluidos en el programa del $85 \%$ de los cursos, mientras que en el $15 \%$ sólo se mencionaron los beneficios estéticos. El $75 \%$ de los cursos se denominaron de inmersión, el $15 \%$ de especialización y el 10\% de perfeccionamiento. El $85 \%$ informó de la carga horaria y, en el 73,68\% de ellos, la duración fue inferior o igual a cinco días. El valor de la hora/clase oscilaba entre 65,00 y 1.000,00 reales. Concluimos que los cursos de armonización orofacial que se ofrecen son de alto valor en comparación con los cursos de otras especialidades y son en su mayoría de corta duración. Además, se comprobó que los proveedores deben adaptar el tipo de curso (actualización, perfeccionamiento, especialización, entre otros) a la respectiva carga de trabajo mínima requerida.

Palabras clave: Odontologia; Estética; Enseñanza.

\section{Introdução}

A busca pela beleza sempre existiu. Ela pode estar atrelada ao ideal de bondade e verdade, como também pode ser vista como algo materializado, objetivamente, na forma de simetria, harmonia, equilíbrio e proporcionalidade (Medeiros, 2011). A beleza parece ser capaz de ampliar o leque de oportunidades de crescimento pessoal e profissional, na medida em que eleva a autoestima e aumenta a segurança do indivíduo (Richter, 2020). E ela nunca se mostrou tão acessível. Resultados que antes só se obtinham por meio de intervenções cirúrgicas invasivas, dolorosas e com longo tempo de recuperação, hoje são atingidos com procedimentos ambulatoriais de execução menos complexa, custo mais baixo e pós-operatório menos debilitante. Basicamente, "se você quer, você pode"; basta fazer o investimento. Por outro lado, os resultados nem sempre são permanentes, demandando a realização de procedimentos adicionais.

O Brasil, que já vinha encabeçando os rankings de cirurgias plásticas realizadas, acabou disparando, também, em procedimentos ligados à harmonização da face, acompanhando a tendência mundial e reiterando o perfil de uma população que sempre valorizou a estética. De acordo com dados da Sociedade Brasileira de Cirurgia Plástica, entre 2014 e 2019 , o número de procedimentos de harmonização facial subiu de 72 mil para 256 mil ao ano. Levando em conta apenas os realizados em homens, o crescimento foi de 255\% (Gaglioni, 2020). Essa situação parece ter se acentuado durante a pandemia da COVID-19. A necessidade de praticar distanciamento tem levado as pessoas a utilizar ainda mais as redes sociais, numa substituição ao convívio presencial. Essa interação vem acontecendo, de maneira síncrona ou não, por meio das telas. É possível que o senso crítico com a própria imagem tenha sido aguçado pelas câmeras dos dispositivos eletrônicos, que captam, refletem e expõem todas as imperfeições (Assis Braga, 2021). Assim, muitos podem ter se tornado reféns da tecnologia de filtros para correção da imagem e também, ter passado a investir mais em procedimentos estéticos. Entre $1^{\circ}$ de março e 14 de junho de 2020 , o faturamento nacional no ramo de cosméticos cresceu 68\%, conforme um estudo da agência Corebiz, de São Paulo (Goeking, 2020). Segundo o Google Trends, ferramenta que monitora as tendências do buscador, as pesquisas com o termo "harmonização facial” cresceram 540\% em 2020, apontando para um amplo e crescente interesse, tanto por parte dos pacientes, que esperam se beneficiar, como por parte dos profissionais, que querem se qualificar para atender a essa demanda (Roebster, 2020). 
Os cirurgiões-dentistas, especialmente de algumas especialidades, costumam ter o senso estético bastante apurado, uma vez que esta é uma habilidade necessária para um profissional que lida, no dia-a-dia, com dimensões milimétricas. Isso os torna aptos à percepção daquela diferença sutil que melhora a harmonia dos traços faciais e se traduz em beleza. Assim, na Odontologia, conhecimentos e princípios vêm sendo aplicados, há tempos, para garantir o resultado estético almejado pelo paciente. Em 2019, por meio da Resolução 198, o Conselho Federal de Odontologia (CFO) reconheceu a "Harmonização Orofacial" (HOF) como especialidade que prevê ao cirurgião-dentista a possibilidade de indicar e realizar vários procedimentos visando o equilíbrio estético, mas também funcional da face, já que a toxina botulínica, por exemplo, também pode ser utilizada no controle da sialorréia, nas dores de cabeça causadas por tensão mastigatória, e também nos quadros clínicos em que incidem hábitos parafuncionais, como bruxismo (da Silva et al., 2020).

Num mercado cada vez mais competitivo, os profissionais da Odontologia passaram a ter, na HOF, uma nova opção de atuação. A especialidade vem conquistando muitos adeptos. Demanda crescente, retorno financeiro, e vasta oferta de cursos formativos têm contribuído para isso. Segundo dados do CFO, o número de especialistas registrados nessa área vem aumentando muito desde 2019, tanto como atuação exclusiva, como em conciliação com outra especialidade, seja por recémformados, como também por profissionais já experientes (CFO, 2019).

Considerando esse cenário e o impacto dos cursos formativos no perfil do profissional, o objetivo do presente trabalho foi caracterizar os cursos de HOF ofertados em um município do Estado do Paraná.

\section{Metodologia}

Trata-se de um estudo observacional transversal (Bonita et al. 2010), cujo objetivo foi descrever as características dos cursos de HOF ofertados, presencial e remotamente, em um município da região Noroeste do Estado do Paraná.

Inicialmente, foi realizado um estudo piloto, com buscas por cursos de HOF na internet em alguns municípios do Paraná, para a avaliação das variáveis de coleta e delimitação da pesquisa. Após a realização de reuniões com os membros da equipe de pesquisadores, foram definidas as variáveis e eleito o município a ser estudado.

A pesquisa foi iniciada com novas buscas de informações em sites de instituições de ensino, redes sociais (Instagram e Facebook) e também na plataforma Google, com as seguintes palavras-chave: "curso de" + "harmonização" ou "harmonização facial" ou "harmonização orofacial" ou "toxina botulínica" ou "botox" ou "preenchimento" ou "ácido hialurônico", limitando a busca para o município estudado. A coleta foi feita no período de 14 a 27 de junho de 2021, e os seguintes dados foram extraídos: nome do curso, instituição, tipo de curso (atualização, aperfeiçoamento, especialização, dentre outros), se era presencial ou à distância, titulação dos coordenadores, carga-horária (teórica, prática e total), duração, número de vagas, custo total, e conteúdo abordado. O valor da hora/aula de cada curso foi calculado a partir da divisão do custo total pelo número de horas do curso, apresentados pela instituição promotora.

Para avaliação da compatibilidade entre carga horária ofertada e tipo de curso, foi observada a regulamentação do Ministério da Educação (MEC) e/ou do Conselho Federal de Odontologia (CFO).

As informações relativas à titulação do coordenador foram obtidas nos sites e perfis de divulgação dos cursos e também por meio de busca na Plataforma Lattes, do CNPq.

\section{Resultados}

A busca resultou em 20 cursos, dos quais 19 eram integralmente presenciais e um era totalmente à distância. As finalidades terapêuticas e estéticas dos procedimentos constavam nos conteúdos programáticos de 17 cursos, enquanto em três havia apenas os objetivos estéticos. Doze eram provenientes de entidades vinculadas a instituições de ensino e oito não informaram a vinculação. 
O Quadro 1 apresenta as características dos cursos ofertados, conforme a categoria. A maioria (75\%) se denominava imersão, $15 \%$ eram especializações e $10 \%$ eram aperfeiçoamentos. Dos três cursos de especialização, apenas um informou a carga horária (555 horas), sendo que a duração variou de 18 a 21 meses. Já nos cursos denominados aperfeiçoamentos, a carga horária média foi de 136 horas, com duração variando de 6 a 10 meses. Os cursos de imersão apresentavam carga horária média de 21 horas e eram realizados em períodos de 1 a 5 dias. Dos 20 cursos analisados, 17 informaram carga horária e, em $73,68 \%$ destes, a duração era menor ou igual a cinco dias.

Dos dois cursos da amostra que se denominavam aperfeiçoamentos, um deles também se intitulou "residência". Considerando a carga horária mínima exigida pela portaria 69/88 do MEC para ambos (aperfeiçoamento e residência), porém, nenhum dos dois cursos se enquadra nas denominações apresentadas.

Quadro 1: Tipo, duração, carga-horária, número de vagas, valor total e valor da hora-aula dos cursos de HOF ( $\mathrm{n}=20$ ).

\begin{tabular}{|c|c|c|c|c|c|c|}
\hline $\begin{array}{l}\text { Cur } \\
\text { so }\end{array}$ & Tipo de Curso & $\begin{array}{l}\text { Duração } \\
\text { (dias/meses) }\end{array}$ & $\begin{array}{l}\text { Carga Horária } \\
\text { (horas) }\end{array}$ & $N^{0}$ de Vagas & $\begin{array}{l}\text { Valor Total } \\
\text { (R\$) }\end{array}$ & $\begin{array}{l}\text { Valor da } \\
\text { Hora/Aula (R\$) }\end{array}$ \\
\hline 1 & Aperfeiçoamento & 10 meses & 152 & $12-24$ & $16.800,00$ & 110,53 \\
\hline 2 & Aperfeiçoamento & 6 meses & 120 & 24 & $9.000,00$ & 75,00 \\
\hline 3 & Especialização & 21 meses & - & $\geq 8$ & $32.550,00$ & - \\
\hline 4 & Especialização & 18 meses & 555 & $\leq 12$ & $36.200,00$ & 65,23 \\
\hline 5 & Especialização & 18 meses & - & - & - & - \\
\hline 6 & Imersão & 5 dias & 56 & $10-24$ & $5.500,00$ & 98,21 \\
\hline 7 & Imersão & 2 dias & 16 & $10-24$ & $2.450,00$ & 153,13 \\
\hline 8 & Imersão & $1 \mathrm{dia}$ & 12 & $10-24$ & $1.350,00$ & 112,50 \\
\hline 9 & Imersão & 3 dias & 24 & - & $3.150,00$ & 131,25 \\
\hline 10 & Imersão & 3 dias & 24 & 10 & $2.620,00$ & 109,17 \\
\hline 11 & Imersão & 1 dia & 8 & 15 & $1.800,00$ & 225,00 \\
\hline 12 & Imersão & 3 dias & - & 15 & $3.000,00$ & - \\
\hline 13 & Imersão & 3 dias & 24 & $\leq 12$ & $3.000,00$ & 125,00 \\
\hline 14 & Imersão & 1 dia & 8 & $\leq 12$ & $8.000,00$ & $1.000,00$ \\
\hline 15 & Imersão & 2 dias & 20 & 10 & $3.150,00$ & 157,50 \\
\hline 16 & Imersão & 1dia & 12 & 10 & $2.500,00$ & 208,33 \\
\hline 17 & Imersão & 3 dias & 30 & 10 & $3.600,00$ & 120,00 \\
\hline 18 & Imersão & 3 dias & 24 & 12 & $3.500,00$ & 145,83 \\
\hline 19 & Imersão & - & 22 & - & 999,99 & 45,45 \\
\hline 20 & Imersão & 3 dias & 30 & - & $2.500,00$ & 83,33 \\
\hline
\end{tabular}

Fonte: Autores (2021). 
O custo total médio de uma especialização foi de $\mathrm{R} \$ 34.375,00$, aperfeiçoamento $\mathrm{R} \$ 12.900,00$ e imersão $\mathrm{R} \$ 3.141,33$. $\mathrm{O}$ valor da hora/aula variou de $\mathrm{R} \$ 65,00$ a $\mathrm{R} \$ 1.000,00$. Dos três cursos de especialização, dois continham, no material de divulgação, as disciplinas da área de concentração e das áreas conexas, incluindo disciplinas obrigatórias.

O Quadro 2 apresenta os dados relativos à formação dos coordenadores. Dois são doutores em ciências odontológicas e nove são mestres em diferentes especialidades, como prótese (1), implantodontia (1), odontopediatria (1), radiologia (2), ortodontia (2), clínica odontológica (1), e DTM (1). Dezesseis coordenadores eram especialistas nas áreas de ortodontia (5), implantodontia (4), odontopediatria (1), cirurgia e traumatologia bucomaxilofacial (1), imaginologia bucomaxilofacial (1), disfunção temporomandibular (1), farmacologia (1), estética facial e corporal (1) e harmonização orofacial (1).

Alguns coordenadores apresentavam também, no currículo, cursos de curta duração nas áreas de implantodontia (1), cirurgia oral menor (1), e harmonização orofacial (1). Um deles não mencionou nenhuma pós-graduação, nem mesmo de curta duração; e outro não possuía currículo cadastrado na plataforma Lattes.

Quadro 2: Formação dos coordenadores dos cursos de HOF da amostra, conforme a área/especialidade e o tipo de pósgraduação.

\begin{tabular}{|c|c|c|c|c|}
\hline Especialidade & $\begin{array}{c}\text { Curso de Curta } \\
\text { Duração }\end{array}$ & Especialização & Mestrado & Doutorado \\
\hline $\begin{array}{c}\text { Harmonização Orofacial } \\
\text { e Estética Corporal e Facial }\end{array}$ & 1 & 2 & & \\
\hline $\begin{array}{c}\text { Especialidades Odontológicas (Prótese, Odontopediatria, } \\
\text { Radiologia, Implantodontia, Ortodontia, Cirurgia e } \\
\text { Traumatologia Bucomaxilo, Disfunção } \\
\begin{array}{c}\text { Temporomandibular, Morfologia em DTM, Ciências } \\
\text { Odontológicas, Clínica Odontológica) }\end{array}\end{array}$ & 2 & 13 & 9 & 2 \\
\hline Outras (Farmacologia) & & 1 & & \\
\hline
\end{tabular}

Fonte: Autores (2021).

\section{Discussão}

Já existem, na literatura, vários estudos sobre uso terapêutico e estético de toxina botulínica, preenchedores faciais, estimuladores de colágeno, bichectomia e outros procedimentos, na Odontologia (Garbin et al., 2019; Lima et al., 2020; Cavalcanti et al., 2017; Machado et al., 2020). Não foram encontrados, porém, estudos caracterizando os cursos de HOF oferecidos no Brasil. Por se tratar de uma especialidade recentemente reconhecida, e considerando o perfil da população brasileira, bem como o interesse crescente por parte de pacientes e profissionais, um estudo dessa natureza pode ser útil para estimular reflexões importantes.

De acordo com o levantamento realizado, dos 20 cursos, apenas três eram de especialização. Os de curta duração (autodenominados “imersão") são os mais ofertados e também os mais procurados porque, por terem carga horária menor, acabam sendo mais acessíveis. Ocorre que, proporcionalmente ao que oferecem, são os que praticam os mais elevados valores de hora/aula, comparados aos de outras áreas da Odontologia. Em geral, são ofertados em instituições, mas também em clínicas e consultórios privados. Faz-se necessário, porém, analisar suas limitações. Segundo a Lei 5.081/66, que regulamenta o exercício da Odontologia no Brasil, no seu artigo $6^{\circ}$, inciso I se verifica que compete ao cirurgião-dentista "praticar todos os atos pertinentes à odontologia, decorrentes de conhecimentos adquiridos em curso regular ou em cursos de pós-graduação”. Pela análise legislativa fica evidente que o conhecimento necessário para praticar um novo ato em clínica só pode ser obtido na graduação ou em cursos de pós-graduação. A Lei 9.394 de 20 de dezembro de 1996, conhecida como Lei de Diretrizes e Bases da Educação brasileira (LDB) define, no seu artigo 44, inciso III, os cursos considerados Pós-Graduação. Não existe menção a cursos de Imersão, na referida norma. O mesmo artigo ainda esclarece que os cursos de Pós-Graduação só devem ser cursados 
por portadores de diploma de graduação, não havendo a prerrogativa para alunos de graduação participarem deste tipo de formação.

Entre as exigências requeridas para realização dos cursos, o Ministério da Educação (MEC), por meio da Portaria 69/88, define 180h e 360h como carga horária mínima para cursos de aperfeiçoamento e especialização, respectivamente. Recomenda-se, porém, que os cirurgiões-dentistas sigam a Resolução 161/2015, do CFO, que define carga horária mínima de especialização conforme as peculiaridades de cada área, variando de 500 a 3.000 horas. Um dos cursos de aperfeiçoamento também se autodenominava "residência". Neste sentido, é preciso esclarecer que os cursos de residência devem ser autorizados pela Comissão Nacional de Residência Multiprofissional em Saúde, e possuir duração e carga horária mínimas de dois anos e 5.760 horas, respectivamente (CFO, 2016). No presente estudo, dois cursos analisados não apresentaram carga horária compatível com o mínimo apontado nem mesmo pela normativa do MEC, para a denominação "aperfeiçoamento", utilizada na divulgação. Considerando as diversas portarias e resoluções, tanto do MEC quanto do CFO, que são esparsas e podem gerar confusão, é possível que as instituições que ofertam os cursos não tenham o conhecimento integral de todas elas. A compreensão das questões normativas relacionadas aos cursos de formação é fundamental, tanto por parte dos proponentes, quanto por parte dos alunos, que poderão fazer escolhas mais criteriosas.

Falando especificamente da HOF, é importante lembrar que a mesma foi reconhecida como especialidade odontológica em 29 de janeiro de 2019, pelo Conselho Federal de Odontologia (CFO), por meio da Resolução 198, a qual também definiu como se daria a atuação dos profissionais. O CFO reconhece como especialista em harmonização orofacial o profissional que tenha realizado curso na área, com carga horária mínima de 500 horas, em instituições credenciadas pelo Conselho ou regulamentadas pelo MEC. Também reconhece como especialista em HOF o já especialista em cirurgia e traumatologia bucomaxilofacial ou outra especialidade registrada, desde que comprove atuação em HOF nos últimos 5 anos. Neste estudo, dentre os 3 cursos de especialização encontrados, somente um informou a carga horária (555h), estando, sim, em conformidade com a exigência mínima do CFO.

Com relação aos limites anatômicos de atuação do cirurgião-dentista, a resolução 176/2016 do CFO determina a região superior ao osso hióide até o ponto násio e, lateralmente, até o tragus da orelha. Também estabelece que procedimentos estéticos, desde que não cirúrgicos, podem ser realizados no terço superior da face. Porém, diante do aumento no número de profissionais atuando em HOF, o CFO publicou, em 2020, uma nova resolução (CFO 230/2020) vetando procedimentos cirúrgicos faciais como alectomia, blefaroplastia, lifting de sobrancelhas, otoplastia, rinoplastia, ritidoplastia, bem como a publicidade e propaganda de procedimentos não odontológicos como micropigmentação de sobrancelhas e lábios, maquiagem definitiva, design de sobrancelhas, remoção de tatuagens faciais, rejuvenescimento de colo e mãos e tratamento de calvície.

A Sociedade Brasileira de Cirurgia Plástica (2021) aponta a toxina botulínica (botox) e o preenchimento como os procedimentos estéticos não cirúrgicos mais procurados no país, na atualidade. Compete ao cirurgião-dentista buscar a qualificação que lhe permitirá ter condições de suprir essa alta demanda. A falta de conhecimento científico e/ou habilidade técnica pode causar danos à saúde e à integridade física do paciente (Nogueira et al. 2014). Como forma de proteger o paciente, a resolução CFO-237/2021 autorizou e regulamentou a aplicação de suspensão cautelar ao cirurgião-dentista que realizar procedimentos, tratamentos e/ou prescrições vedados ou não reconhecidos como exercício da odontologia; que ultrapassar os limites da competência legal da profissão; que praticar ou acobertar exercício ilegal da profissão; e que realizar, ministrar, patrocinar ou divulgar cursos vedados ou não reconhecidos.

Em relação à formação dos coordenadores dos cursos, apenas dois não informaram ter pós-graduação stricto sensu ou lato sensu em Odontologia. Esses profissionais, entretanto, estavam à frente de cursos de curta duração. Segundo o CFO (2019), o coordenador de curso de especialização é que precisa ter, no mínimo, pós-graduação stricto sensu, devendo o corpo docente da área de concentração ser composto, exclusivamente, por no mínimo especialistas na área, devidamente registrados 
no CFO. Não há exigências para os cursos de curta duração. O CFO possui referência normativa própria apenas para os cursos de especialização e para as habilitações em Odontologia, sendo que estas últimas não se aplicam ao trabalho em questão. De qualquer forma, as informações disponibilizadas sobre coordenadores e professores podem ser úteis no momento da escolha do curso.

Não há dúvida de que o cirurgião-dentista, ao realizar procedimentos estéticos exitosos, contribui para a saúde emocional do indivíduo (Papazian et al. 2018). A própria Organização Mundial da Saúde (OMS) define "saúde" como sendo não apenas a ausência de doença, mas também um estado de completo bem-estar que envolve os contextos físico, emocional, mental e social (OMS, 1946). A grande preocupação, porém, com o aumento na oferta e na procura por procedimentos estéticos, é que as necessidades básicas em saúde bucal sejam deixadas de lado. Atualmente, no país, dos 362.303 cirurgiõesdentistas, 799 são especialistas em HOF, mas esse número está crescendo rapidamente (CFO, 2021; CFO, 2021b), e o país segue apresentando altos índices de doenças bucais, incluindo cárie e doença periodontal (Brasil, 2012). A integralidade na avaliação da saúde bucal deve ser priorizada pelo profissional da Odontologia. Ciente de suas necessidades de diferentes ordens, o paciente poderá, então, fazer suas escolhas.

A lógica é que um indivíduo busque a resolução de problemas estéticos somente quando lhe sobrem recursos após sanados os problemas que prejudicam a vida ou tolhem a função. É a priorização da saúde, como necessidade, em detrimento da estética, algo desejável, mas não essencial. Essa lógica, porém, nem sempre se aplica. Conforme a Pesquisa de Endividamento e Inadimplência do Consumidor (Peic), realizada pela Confederação Nacional do Comércio de Bens, Serviços e Turismo (CNC), o $1^{\circ}$ semestre de 2021 terminou com 69,7\% das famílias brasileiras endividadas: uma alta de 2,5\% em comparação a junho de 2020. Um levantamento realizado pelo Serviço de Proteção ao Crédito (SPC) mostrou, em junho de 2016, que o consumo de serviços e produtos de beleza normalmente se dá por impulso; que 49,4\% dos brasileiros acreditam que melhorar a aparência física é um investimento que vale a pena, por proporcionar sensação de felicidade e satisfação; que $11 \%$ deixam de cumprir compromissos financeiros para priorizar a beleza; e que 39\% preferem adquirir produtos ou serviços de natureza estética em vez de economizar. Os brasileiros parecem se desorganizar financeiramente, confundindo necessidade com desejo e se deixando levar pelo ideal de beleza que lhes é apresentado, diariamente, nos meios de comunicação e nas mídias sociais.

\section{Conclusão}

Conclui-se que os cursos de harmonização orofacial da amostra são, em sua maioria, de curta duração e o investimento requerido por parte dos interessados é alto, comparativamente a outras especialidades.

Como alerta, temos que:

1. as entidades promotoras poderiam adequar o tipo de curso (atualização, aperfeiçoamento, especialização, dentre outros) à respectiva carga-horária mínima exigida;

2. os profissionais poderiam escolher o curso usando como critérios o conteúdo programático, a formação do coordenador e dos professores, como também a compatibilidade entre carga horária oferecida e a sua própria necessidade formativa (cursos de curta duração para os experientes, e cursos de especialização para os que vão iniciar na área);

3. os profissionais deveriam primar pelo cuidado integral à saúde do paciente (física, emocional, mental, social e também financeira), inclusive para melhor auxiliá-lo no estabelecimento de prioridades;

4. se a especialidade HOF for exercida de forma devidamente respaldada, tanto técnica como cientificamente, a classe odontológica, como um todo, sairá fortalecida. 
Os resultados deste estudo são significativos, porém algumas limitações precisam ser ressaltadas, como a amostra reduzida, a ausência de estudos prévios, e a dificuldade na obtenção de determinadas informações, visto que a maior parte da extração foi feita a partir de material de divulgação. Seria interessante que mais estudos fossem realizados, em diferentes municípios e com amostras maiores.

\section{Referências}

Bonita, R., Beaglehole R., \& Kjellstrom T. (2010) Epidemiologia Básica. Santos.

Brasil. (1966). Lei $n^{\circ} 5.081$, de 24 de agosto de 1966. http://www.planalto.gov.br/ccivil_03/leis/15081.htm.

Brasil. (1996). Lei $n^{\circ} 9.394$, de 20 de dezembro de 1996. http://www.planalto.gov.br/ccivil_03/leis/19394.htm.

Brasil. Ministério da Educação, Conselho Nacional de Educação, Câmara de Educação Superior. (2007). Resolução $n^{\circ} 1$, de 8 de junho de 2007. http://portal.mec.gov.br/index.php?option=com_docman\&view=download\&alias=8825-rces001-07-pdf\&category_slug=setembro-2011-pdf\&Itemid=30192.

Brasil. Ministério da Saúde. Secretaria de Atenção à Saúde. Secretaria de Vigilância em Saúde. (2010). SB Brasil 2010: Pesquisa Nacional de Saúde Bucal: resultados principais. https://bvsms.saude.gov.br/bvs/publicacoes/pesquisa_nacional_saude_bucal.pdf.

Conselho Federal de Odontologia. (2020). Resolução CFO-230, de 14 de agosto de 2020.

https://sistemas.cfo.org.br/visualizar/atos/RESOLU\%C3\%87\%C3\%83O/SEC/2020/230.

Conselho Federal de Odontologia. (2019). Resolução CFO-195, de 29 de janeiro de 2019.

https://sistemas.cfo.org.br/visualizar/atos/RESOLU\%c3\%87\%c3\%83O/SEC/2019/195.

Conselho Federal de Odontologia. (2019). Resolução CFO-197, de 29 de janeiro de 2019. https://sistemas.cfo.org.br/visualizar/atos/RESOLU\%c3\%87\%c3\%83O/SEC/2019/197.

Conselho Federal de Odontologia. (2021). Resolução CFO-237/2021. http://www.crodf.org.br/pdf/resolucaocfo-237-2021.pdf.

Conselho Federal de Odontologia. (2019). Resolução CFO-198, de janeiro de 2019. https://sistemas.cfo.org.br/visualizar/atos/RESOLU\%C3\%87\%C3\%83O/SEC/2019/198.

Conselho Federal de Odontologia. (2012). Resolução do Conselho Federal de Odontologia - CFO 118 de 11.05.2012. http://www.normaslegais.com.br/legislacao/resolucao-cfo-118-2012.htm.

Conselho Federal de Odontologia. (2015). Resolução CFO-161/2015. https://website.cfo.org.br/wp-content/uploads/2015/11/Resolu\%C3\%A7\%C3\%A3oCFO-161-15-nova-espcialidade-II.pdf.

Conselho Federal de Odontologia. (2021). Quantidade geral de profissionais e entidades ativas. https://website.cfo.org.br/estatisticas/quantidade-geral-deentidades-e-profissionais-ativos/.

Conselho Federal de Odontologia. (2021b). Quantidade geral de cirurgiões-dentistas especialistas. https://website.cfo.org.br/estatisticas/quantidade-geral-decirurgioes-dentistas-especialistas/.

Cavalcanti, A. N., Azevedo, J.F. \& Mathias, P. (2017). Harmonização Orofacial: A Odontologia além do sorriso. Revista Bahiana de Odontologia. 2017 June;8(2):35-36. DOI: 10.17267/2238-2720revbahianaodonto.v8i2.1454.

Confederação Nacional do Comércio de Bens, Serviços e Turismo. (2021). Forte alta no endividamento: 7 em cada 10 famílias encerram o semestre com dívidas. Endividamento e Inadimplência do consumidor. https://portal-bucket.azureedge.net/wp-content/2021/07/Analise_Peic_junho_2021.pdf

de Medeiros, A. C. (2011). O ideal de beleza na escultura grega: reflexões sobre as acepções formais construídas pela sociedade grega. PRINCIPIA, (23), 89102.

da Silva, A. A., Nepomuceno Filho, D. G., Bomfim, L. M., Silva, L. A. M., da Cunha, M. S., de Albuquerque, S. C., \& Neto, J. F. T. (2020). Indicações para fins terapêuticos da toxina botulínica do tipo A no uso odontológico: uma revisão de literatura. Revista Eletrônica Acervo Saúde, (58), e4348-e4348.

de Assis Braga, M. L., de Almeida, I. F., Borges, F. D. S. Q., Feitosa, H. A., Costa, L. E. D., \& Feitosa, F. D. S. Q. (2021). Avaliação da percepção de satisfação do sorriso e da influência das mídias sociais digitais na população. Research, Society and Development, 10(6), e46810615727-e46810615727.

Gaglioni, C. (2020). O que é harmonização facial. E como ela cresce no Brasil. https://www.nexojornal.com.br/expresso/2020/09/29/O-que-\%C3\%A9harmoniza\%C3\%A7\%C3\%A3o-facial.-E-como-ela-cresce-no-Brasil

Garbin, A. J. I., Wakayama, B., Saliba, T.A. \& Garbin, C.A.S. (2019). Harmonização Orofacial e suas implicações na Odontologia. Brazilian Journal of Surgery and Clinical Research - BJSCR, 27(2), 116-122.

Goeking, W. (2020). Faturamento do comércio eletrônico de cosméticos cresce 68\% durante quarentena. https://valorinveste.globo.com/mercados/rendavariavel/empresas/noticia/2020/06/26/faturamento-do-comercio-eletronico-de-cosmeticos-cresce-68percent-durante-quarentena.ghtml.

Lima, N. B. \& Soares, M. L. (2020). Utilização dos bioestimuladores de colágeno na harmonização orofacial. Clin Lab Res Den 2020:1-18. http://dx.doi.org/10.11606/issn.2357-8041.clrd.2020.165832. 
Research, Society and Development, v. 10, n. 17, e242101724660, 2021

(CC BY 4.0) | ISSN 2525-3409 | DOI: http://dx.doi.org/10.33448/rsd-v10i17.24660

Machado, L. L. Atuação do cirurgião dentista na harmonização orofacial. (Dissertação, Hospital de clínicas de Porto Alegre, programa de pós-graduação Mestrado Profissional em Pesquisa Clínica). https://lume.ufrgs.br/bitstream/handle/10183/214031/001117885.pdf?sequence=1\&isAllowed=y.

Ministério da Educação e Conselho Nacional de Educação. http://portal.mec.gov.br/cne/arquivos/pdf/pces263_06.pdf

Morita, A. (2020). Harmonização facial cresce cada vez mais no Brasil e no mundo. https://www.terra.com.br/noticias/dino/harmonizacao-facial-cresce-cadavez-mais-no-brasil-e-no-mundo,37419eb012595ad25e3a9f6be429f05dtrm0tzud.html.

Organização Mundial da Saúde (1946). Constituição da Organização Mundial da Saúde (OMS/WHO).

Papazian, M. F., Silva, L. M., Crepaldi, A. A., Crepaldi, M. L. S., \& Aguiar, A. P. (2018). Principais aspectos dos preenchedores faciais. Revista FAIPE, 8(1), 101-116.

Richter, L. L. (2020). A estética como empoderamento da mulher líder.

Roebster, C. (2020). Pretty Hurts: a busca da perfeição através de procedimentos estéticos. https://primeirapauta.ielusc.br/2020/12/19/pretty-hurts-a-buscada-perfeicao-atraves-de-procedimentos-esteticos/.

Serviço de Proteção ao Crédito. (2016). Significados da beleza: autoimagem e consumo.

https://www.spcbrasil.org.br/wpimprensa/wp-content/uploads/2016/06/Apresentacao_mercado_beleza-1.pdf.

Sociedade Brasileira de Cirurgia Plástica. Harmonização facial: o que é e por que está tão em alta.

http://www2.cirurgiaplastica.org.br/2021/01/28/harmonizacao-facial-o-que-e-e-por-que-esta-tao-em-alta/. 